\title{
A multi-moment transport model on cubed-sphere grid
}

\author{
C. G. Chen ${ }^{1, * \dagger}$, F. Xiao ${ }^{1,2}$, X. L. Li ${ }^{3}$ and Y. Yang ${ }^{1}$ \\ ${ }^{1}$ LHD, Institute of Mechanics, Chinese Academy of Sciences, Beijing, People's Republic of China \\ ${ }^{2}$ Department of Energy Sciences, Tokyo Institute of Technology, Yokohama, Japan \\ ${ }^{3}$ Center of Numerical Weather Prediction, Chinese Meteorology Administration, Beijing, \\ People's Republic of China
}

\begin{abstract}
SUMMARY
A conservative, single-cell-based semi-Lagrangian transport model is proposed in this paper. Using multimoment concept, an additional moment, i.e. volume-integrated average (VIA), is treated as the model variable besides the point value (PV) updated in the traditional semi-Lagrangian schemes. A quadratic interpolation function is constructed based on local degrees of freedom defined within each single cell. The PV moment is advanced by the semi-Lagrangian formulation, whereas the VIA moment is updated by a finite volume formulation to rigorously ensure the numerical conservation. The numerical fluxes are computed from the PV moments defined along the boundary edges of the control volume. The scheme is extended to the spherical geometry through the application of the cubed-sphere grid that eliminates the polar singularity in the conventional longitude/latitude coordinates by using the quasi-uniform grid spacing covering the whole sphere. The single-cell-based scheme is well suited for the treatment of the connections between different patches. A simple quasi-monotone limiter to the PV moment is applied to suppress non-physical oscillations. The proposed scheme has been validated via representative benchmark tests and the performance is competitive to other existing transport schemes. Copyright (C) 2010 John Wiley \& Sons, Ltd.
\end{abstract}

Received 23 March 2010; Revised 20 September 2010; Accepted 29 September 2010

KEY WORDS: $\quad$ semi-Lagrangian scheme; finite volume method; transport process; global model; spherical geometry; cubed-sphere grid

\section{INTRODUCTION}

Computation of the trace constituent transport is critical to the general circulation models (GCMs). It is not a trivial work to develop numerical transport model of adequate accuracy and efficiency, especially in the spherical geometry which is in general involved in the global GCMs. The conventional longitude/latitude (LON/LAT) grid has singularity at two poles. Moreover the convergence of the meridians in the polar region gives rise to a serious reduction in the mesh spacing, and thus the time stepping due to the CFL stability condition. Semi-Lagrangian schemes have gained popularity in constructing the transport model on the LON/LAT grid because of the larger allowable CFL number for computational stability. As reviewed in [1], the semi-Lagrangian schemes are able to produce accurate results even for considerably large time steps. One of the major drawbacks of the semi-Lagrangian schemes, however, is that they are not inherently conservative. The conservation error is particularly important and fatal in some cases for long-term simulations.

Many efforts have been made to develop the conservative semi-Lagrangian schemes. Among others, a class of conservative semi-Lagrangian schemes, which follow the underlying concept of

\footnotetext{
${ }^{*}$ Correspondence to: C. G. Chen, LHD, Institute of Mechanics, CAS, No. 15 Beisihuanxi Road, Haidian District, Beijing 100190, People's Republic of China.

†E-mail: cgchen@imech.ac.cn
} 
the CIP (Cubic Polynomial Interpolation [2] or more generally Constrained Interpolation Profile [3]) method and are so-called CIP (Constrained Interpolation Profile) Conservative Semi-Lagrangian (CIP-CSL) schemes, were proposed in [4-8] by introducing multi moments $[9,10]$ as the computational variables. A convenient and efficient transport model with numerical conservation can be constructed by making use of two kinds of moments, i.e. point value (PV moment) and volumeintegrated average (VIA moment). PV moment can be predicted by the normal semi-Lagrangian schemes, whereas the VIA moment is updated by the flux-form finite volume formulation and thus is exactly conserved. The original CIP-CSL schemes use the rigorous integration of the interpolation function to obtain the numerical flux, and meet substantial complexity when one tries to integrate an interpolation function in higher dimensions in two-dimensional or three-dimensional applications where a dimensional splitting is often implemented as a practical way. In this paper, we report a more flexible extension of the semi-Lagrangian multi-moment advection schemes by using high-order quadrature along the trajectory for the computation of the numerical flux. The present method can be straightforwardly implemented to multi-dimensional computation without the dimensional splitting. It also should be pointed out that some conservative semi-Lagrangian transport models $[11,12]$ have been constructed through the rigorously integrating spatial reconstruction polynomials although the numerical procedure is very complicated.

Another important part of this paper is to build up a practical numerical formulation for advection transport in spherical geometry using quasi-uniform mesh. The efforts to represent the sphere with quasi-uniform grid spacing, in fact, started more than 40 years ago. Two representatives of the non-LON/LAT grids, i.e. icosahedron grid [13,14] and cubic grid [15], were used to solve barotropic models on sphere. Owing to the complex structures of the these grids, however, they had not gotten a wide popularity until the practice to implement some modern sophisticated numerical methods in the past decade. The recent success with these uniformly spaced spherical grids has made them widely accepted in developing next generation global models for geophysical flows.

The icosahedron grid possesses a more uniform grid spacing compared with the cubic grid, but involves significant complexity and requires numerical techniques considerably different from those developed for the structured grids. Contrarily, the cubic grid projects the surface of the sphere onto an inscribed cube and the computational domain is mapped to six square patches where structured grid topologically equivalent to the Cartesian one is constructed. Some numerical models have been developed on cubic grid for both transport and shallow-water flows as given in $[12,16-25]$. On cubic grid, the curvilinear coordinates within each patch can be constructed by a gnomonic projection [15] or a conformal projection [20]. The gnomonic projection has more uniform grid spacing, but encounters the broken coordinates along the boundary edges between different patches. It brings about difficulties to communicate data from one patch to another. Extra numerical manipulations are required for the computations over the stencils next to the boundary edges. The conformal projection method is proposed to overcome this difficulty, but at a cost of losing the uniformity of the grid and analytic transformation laws. In our former work [26], a global shallow-water model has been constructed on cubed-sphere grid using multi-moment concept. Different from the original CIP-CSL schemes, the PV moment is computed by solving a derivative Riemann problem (DRP) at cell interface and time integration was implemented by the Runge-Kutta scheme. Under this numerical framework, we can conveniently treat the forcing terms of shallow-water equations.

As an efficient semi-Lagrangian advection transport model in spherical geometry, we present in this paper a CIP-type multi-moment conservative scheme on cubed-sphere grid. The major difference from the existing CIP-CSL schemes [4-8] is that the numerical flux in the present scheme is computed from the values sampled along the semi-Lagrangian trajectory instead of the rigorous integration of the interpolation function, and only two kinds of moments, i.e. PV and VIA, are used as the computational variables even in multi-dimensional cases. The present formulation is well suited for the cubed-sphere grid, where the data transfer is required on the patch boundaries. Especially, in the present model the single-cell quadratic reconstruction based on the local degrees of freedom (DOFs) of multi moments minimizes the ghost cells required for inter-patch data communication. 
The remainder of this paper is organized as follows. The basic formulation of semi-Lagrangian multi-moment transport scheme for one-dimensional case is described in Section 2 with numerical tests. The scheme on two-dimensional Cartesian grid is given in Section 3 and extended to spherical geometry with the application of cubed-sphere grid in Section 4 . We evaluate the proposed scheme with some representative benchmark numerical experiments in Section 5. A few concluding remarks are given in Section 6.

\section{BASIC FORMULATION IN ONE DIMENSION}

To build a multi-moment scheme, the first step is to decide the moment configuration. As shown in Figure 1, we consider the one-dimensional control volume $\mathscr{C}_{i} \equiv\left[x_{i-\frac{1}{2}}, x_{i+\frac{1}{2}}\right]$. Without losing generality, the uniform grid spacing $\Delta x$ is adopted here. The PV moment and VIA moment of the transported field $\phi(x, t)$ are defined as

- The PV moment

$$
\bar{P}_{i \pm \frac{1}{2}}(t)=\phi\left(x_{i \pm \frac{1}{2}}, t\right) \text { at the endpoints of } \mathscr{C}_{i},
$$

- The VIA moment over $\mathscr{C}_{i}$

$$
\bar{V}_{i}(t)=\frac{1}{\Delta x} \int_{x_{i-\frac{1}{2}}}^{x_{i+\frac{1}{2}}} \phi(x, t) \mathrm{d} x .
$$

Then we build the spatial reconstruction with the two moments defined above. With two PV moments and one VIA moment in each control volume, a piecewise quadratic interpolation function (same as that used in the CIP-CSL2 scheme [4]) can be constructed piecewisely for cell $i$ as

$$
\Phi_{i}(x)=c_{i}^{0}+c_{i}^{1} \tilde{x}+c_{i}^{2} \tilde{x}^{2},
$$

where $\tilde{x}=x-x_{i-1 / 2}$.

By solving the constraints in terms of both PV and VIA moments as

$$
\begin{aligned}
& \bar{P}_{i \pm \frac{1}{2}}=\Phi_{i}\left(x_{i \pm \frac{1}{2}}\right), \\
& \bar{V}_{i}=\frac{1}{\Delta x} \int_{x_{i-\frac{1}{2}}}^{x_{i+\frac{1}{2}}} \Phi_{i}(x) \mathrm{d} x,
\end{aligned}
$$

the coefficients read as

$$
\begin{aligned}
c_{i}^{0} & =\bar{P}_{i-\frac{1}{2}}, \\
c_{i}^{1} & =-\frac{2}{\Delta x}\left(2^{P} \bar{\phi}_{i-\frac{1}{2}}+\bar{P}_{i+\frac{1}{2}}-3^{V} \bar{V}_{i}\right), \\
c_{i}^{2} & =\frac{3}{\Delta x^{2}}\left(\bar{P}_{i-\frac{1}{2}}+\bar{P}_{i+\frac{1}{2}}-2^{V} \bar{V}_{i}\right) .
\end{aligned}
$$

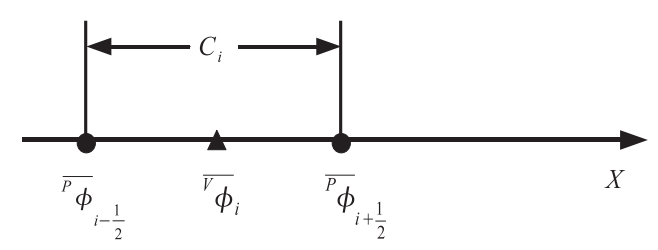

Figure 1. Moment configuration for one-dimensional case. 
The governing equation for one-dimensional transport of physical field $\phi(x, t)$ is written as

$$
\frac{\partial \phi}{\partial t}+\frac{\partial(u \phi)}{\partial x}=0
$$

The PV moment is updated by using the semi-Lagrangian scheme. The governing equation is recast into advection form using substantial derivative as

$$
\frac{\mathrm{D} \phi}{\mathrm{D} t}=-\phi \frac{\partial u}{\partial x} \text {. }
$$

Provided the PV moment and VIA moment at time $t^{n}$, i.e. $\bar{P}_{i \pm 1 / 2}^{n}$ and $\bar{V}_{i}^{n}$ for the $i$ th cell, the reconstruction polynomial $\Phi_{i}^{n}(x)$ in (3) is found with the coefficients given in (5). Considering the problems with constant speed, the right-hand side term of (7) vanishes. According to the Lagrangian invariant solution, we obtain the PV moment at the next time step as

$$
\bar{P}_{i-\frac{1}{2}}^{n+1}=\Phi_{I}^{n}\left(x_{i-\frac{1}{2}}^{P}\right)
$$

where $x_{i-1 / 2}^{P}$ is the location of the departure point corresponding to grid point $x_{i-1 / 2}$ at $t=t^{n}$, which can be simply computed by $x_{i-1 / 2}^{P}=x_{i-1 / 2}-u \Delta t$ in the case where the advecting velocity is constant. The subscript $I$ means the index of the cell in which the departure point $x_{i-1 / 2}^{P}$ falls in.

For the problems with variable speed, the right-hand side term of (7) is considered as a source term and should be integrated along the trajectory. The departure point should be calculated by time integration as $x_{i-1 / 2}^{P}=x_{i-1 / 2}-\int_{t^{n}}^{t^{n+1}} u(x) \mathrm{d} t$, where some high-order numerical schemes for ODE can be used, e.g. the multi-step Runge Kutta scheme described later in this paper.

By integrating (6) over the $i$ th element, the VIA moment can be updated using the formulation of

$$
\frac{\partial^{V} \bar{\phi}_{i}}{\partial t}=-\frac{1}{\Delta x}\left(f_{i+\frac{1}{2}}-f_{i-\frac{1}{2}}\right)
$$

where $f=u \phi$ is the flux function. This finite volume formulation guarantees the numerical conservation of the VIA moment.

To solve the ODE (9), the time integration of the numerical flux can be computed by analytically integrating the interpolation function along the trajectory as in the original CIP-CSL schemes $[4,5]$,

$$
g_{i-\frac{1}{2}}=\int_{t^{n}}^{t^{n+1}} f_{i-\frac{1}{2}} \mathrm{~d} t= \begin{cases}\Delta x \bar{V}_{I}-\int_{x_{I-\frac{1}{2}}}^{x^{P}}{ }^{P-\frac{1}{2}} \Phi_{I}(x) \mathrm{d} x+\Delta x \sum_{p=I+1}^{i-1} \bar{V}_{p} & \text { if } u>0, \\ -\int_{x_{I-\frac{1}{2}}}^{x_{i-\frac{1}{2}}^{P}} \Phi_{I}(x) \mathrm{d} x-\Delta x \sum_{p=i}^{I-1} \bar{V}_{p} & \text { if } u<0 .\end{cases}
$$

However, it is very complicated and costly, even possible, to get a rigorous integration of the interpolation function over a multi-dimensional area in two- or three-dimensional cases. An alternative method used by Ii and Xiao [27] to evaluate the numerical flux is to compute the numerical quadrature in terms of the values sampled along the trajectory by an explicit multi-point integration scheme, e.g. $M$-point Simpson's rule, as

$$
g_{i-\frac{1}{2}}=\Delta \sum_{p=1}^{M} \beta_{p} \Phi_{I_{p}}\left(x_{i-\frac{1}{2}}-\alpha_{p} \Delta\right),
$$

where $\Delta=x_{i-1 / 2}-x_{i-1 / 2}^{P}, I_{p}$ is the index of the cell in which the $p$ th sampled point with the location of $x_{i-1 / 2}-\alpha_{p} \Delta$ falls in. When a quadratic interpolation function is adopted in spatial reconstruction, one arrives at the 3-point Simpson's quadrature formula. Thus, the coefficients are

$$
\alpha_{1}=0, \quad \alpha_{1}=\frac{1}{2}, \quad \alpha_{2}=1 \quad \text { and } \quad \beta_{1}=\beta_{3}=\frac{1}{6}, \quad \beta_{2}=\frac{2}{3} .
$$


In case of constant speed, numerical flux in (11) is computed by

$$
g_{i-\frac{1}{2}}=\Delta t \sum_{p=1}^{M} \beta_{p} f_{i-\frac{1}{2}}\left(t^{n}+\alpha_{p} \Delta t\right)=\Delta t \sum_{p=1}^{M} \beta_{p} u_{i-\frac{1}{2}} \bar{P}_{i-\frac{1}{2}}\left(t^{n}+\alpha_{p} \Delta t\right),
$$

where $\bar{P}_{i-1 / 2}\left(t^{n}+\alpha_{p} \Delta t\right)$ is the value of PV moment at the time of $t^{n}+\alpha_{p} \Delta t$, which is calculated by the same semi-Lagrangian scheme mentioned above using $\alpha_{p} \Delta t$ as the time increment.

Once $g_{i-1 / 2}$ is obtained at the interfaces of all cells, the VIA moment at $t^{n+1}$ is advanced by

$$
\bar{V}_{i}^{n+1}=\bar{V}_{i}^{n}-\frac{1}{\Delta x}\left(g_{i+\frac{1}{2}}-g_{i-\frac{1}{2}}\right) .
$$

Being a semi-Lagrangian type, the present scheme works stably even with large CFL as in the original CIP-CSL schemes [4,5], where the common parts of the fluxes passing through $x_{i-1 / 2}$ and $x_{i+1 / 2}$ are canceled out in the numerical integration. However, it should be notified that the computational stability is somewhat dependent on the order of the numerical quadrature for the flux function in the present scheme. The Von Neumann analysis given in Appendix A shows that a 3-point Simpson's rule requires the all sampled points to be within the mesh cell containing the departure point, which means that the local CFL number $\left(\mathrm{CFL}_{\mathrm{loc}}\right)$ must satisfy $\mathrm{CFL}_{\mathrm{loc}}<1$, and the 5-point Simpson's quadrature is stable where even the sampled points spread over more than one cell and thus allows a larger $\mathrm{CFL}_{\mathrm{loc}}$ for stability.

Same as other high-resolution schemes, in the presence of discontinuity or large gradient, a properly chosen limiter is necessary to suppress the non-physical oscillations. A simple limiter proposed in [28] is used in the present study, i.e. after being updated from the Lagrangian invariant solution (8), the PV moments should be limited by the local extremes. We set the extremes for the $i$ th cell as the maximal and minimal PV moments

$$
\phi_{i}^{+}=\max \left(\bar{P}_{i \pm \frac{1}{2}}^{n}\right) \quad \text { and } \quad \phi_{i}^{-}=\min \left(\bar{P}_{i \pm \frac{1}{2}}^{n}\right) .
$$

The PV moment at time step $t^{n+1}$ is limited to be

$$
\bar{P}_{i-\frac{1}{2}}^{n+1}=\max \left\{\phi_{I}^{-}, \min \left[\phi_{I}^{+}, \Phi_{I}^{n}\left(x^{P}\right)\right]\right\} .
$$

It should be pointed out that we only apply the limiter to the PV moment here. It guarantees the oscillation-less results for PV moment, at the same time, as can be seen from the numerical results the non-physical oscillations of VIA moment can be effectively controlled as well. It should be noted that this limiting procedure does not cause any detriment to the conservation of the VIA moment.

\section{Example 2.1}

We use this numerical test to evaluate the convergence rate of the scheme discussed above. The initial condition is given by

$$
\phi(x, 0)=\sin (\pi x), \quad x \in[-1,1]
$$

with a constant speed $u=1$.

The numerical experiments are carried out on gradually refined grids with the CFL number fixed at $0.25,0.8$ by the 3 -point scheme and 1.25 by the 5-point scheme. The accuracy of the present scheme is examined by the normalized $l_{2}$ error defined in [29]. The numerical errors and the convergence rate are shown in Table I. The third-order accuracy is achieved as expected. In practical use, the number of sampled points along trajectory for numerical quadrature of flux is problem-dependent and usually determined on a trade-off between computational accuracy and cost. In this paper, we only give the numerical results by 3-point scheme hereafter for the sake of brevity. 
Table I. Convergence test for one-dimensional case.

\begin{tabular}{|c|c|c|c|c|c|c|}
\hline \multirow[b]{2}{*}{ Grid } & \multicolumn{2}{|c|}{$\mathrm{CFL}=0.25$} & \multicolumn{2}{|c|}{$\mathrm{CFL}=0.8$} & \multicolumn{2}{|c|}{$\mathrm{CFL}=1.25$} \\
\hline & $l_{2}$ error & $l_{2}$ order & $l_{2}$ error & $l_{2}$ order & $l_{2}$ error & $l_{2}$ order \\
\hline 20 & $1.6302 \mathrm{e}-3$ & - & $4.4347 \mathrm{e}-4$ & - & $3.3696 \mathrm{e}-4$ & - \\
\hline 40 & $2.0536 \mathrm{e}-4$ & 2.99 & $5.6217 \mathrm{e}-5$ & 2.98 & $4.3219 \mathrm{e}-5$ & 2.96 \\
\hline 80 & $2.5729 \mathrm{e}-5$ & 3.00 & $7.0679 \mathrm{e}-6$ & 2.99 & $5.4584 \mathrm{e}-6$ & 2.99 \\
\hline 160 & $3.2187 e-6$ & 3.00 & $8.8576 e-7$ & 3.00 & $6.8537 e-7$ & 2.99 \\
\hline 320 & $4.0246 e-7$ & 3.00 & $1.1085 \mathrm{e}-7$ & 3.00 & $8.5848 \mathrm{e}-8$ & 3.00 \\
\hline
\end{tabular}
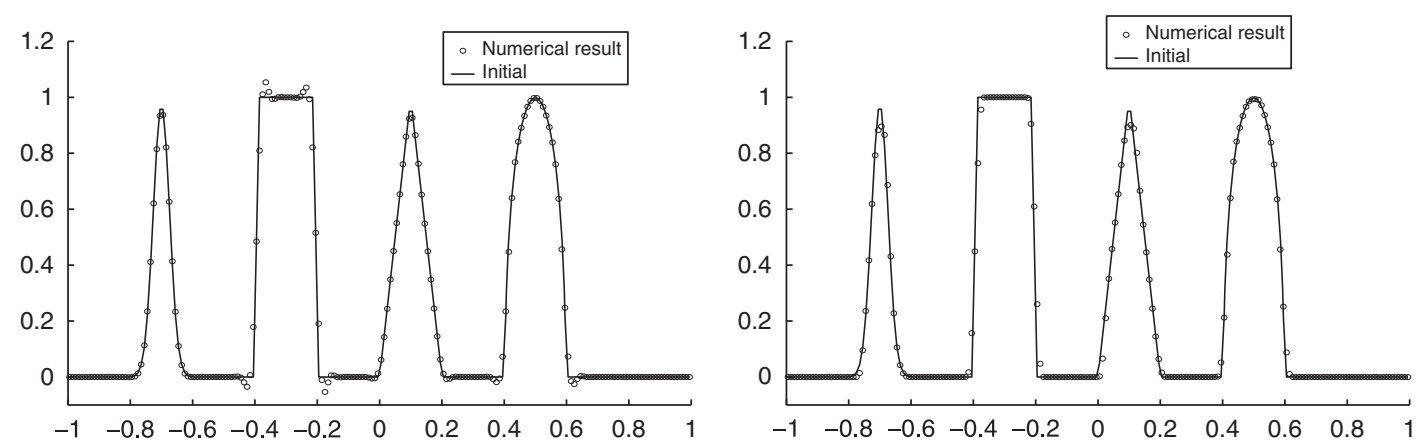

Figure 2. One-dimensional advection test with a composite initial distribution. Shown are the result without limiter (left) and the result with PV limiter (right).

\section{Example 2.2}

A one-dimensional test with complex initial distribution including both smooth and discontinuous parts is computed. The transported field consists of a Gaussian distribution, a square wave, a sharp triangle and a half ellipse. The detailed expressions can be found in [30]. The computational domain $(x \in[-1,1])$ is divided into 200 cells, the CFL number is specified as 0.8 . We ran the numerical test for four revolutions, respectively, with and without the numerical limiter. The numerical result without limiting is shown in the left panel in Figure 2. Obvious oscillations are observed. The overshooting and undershooting errors in the numerical solution of the VIA moment are $5.3617 \times 10^{-2}$ and $-5.3617 \times 10^{-2}$, respectively. The numerical result with the limiting projection (16) onto the PV moment is shown in the right panel in Figure 2. The overshooting and undershooting errors in the PV moment are completely eliminated, and those in the VIA moment are significantly reduced to $4.6152 \times 10^{-4}$ and $-5.4457 \times 10^{-4}$, an acceptable extent to many practical applications. It reveals that the simple limiter used in the present scheme effectively controls the spurious oscillations while keeping the accuracy of the numerical schemes. We have got at the same time the numerical solution to PV moment completely free from numerical oscillation and an exactly conserved solution to VIA moment.

\section{EXTENSION TO TWO-DIMENSIONAL CARTESIAN GRID}

To construct a single-cell-based piecewise quadratic reconstruction polynomial for two-dimensional rectangular control volume, nine DOFs are required [26]. The moment configuration for the transported field $\phi(\mathbf{x}, t)$ on two-dimensional control volume $\mathscr{C}_{i j}\left(\left[x_{i-1 / 2}, x_{i+1 / 2}\right] \times\left[y_{j-1 / 2}, y_{j+1 / 2}\right]\right)$ is shown in Figure 3. Four PV moments are located at the vertices of the control volume, two PV moments are defined at the midpoints of the $x$-direction edges and other two at the midpoints of 


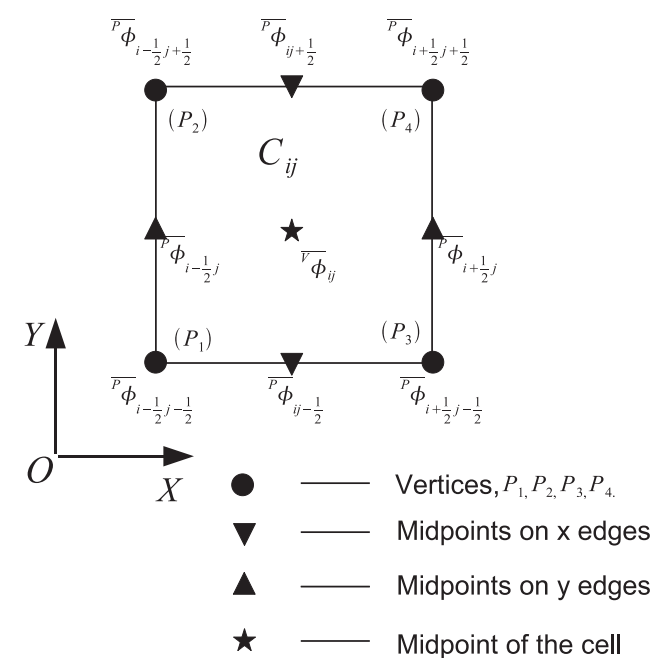

Figure 3. Moment configuration for two-dimensional case.

the $y$-direction edges,

$$
\begin{array}{ll}
\bar{P}_{i \pm \frac{1}{2} j \pm \frac{1}{2}}(t)=\phi\left(x_{i \pm \frac{1}{2}}, y_{j \pm \frac{1}{2}}, t\right) & \text { at vertices, } \\
\bar{P}_{i j \pm 1 / 2}(t)=\phi\left(x_{i}, y_{j \pm \frac{1}{2}}, t\right) & \text { at midpoints of } x \text {-direction edges, } \\
\bar{P}_{i \pm \frac{1}{2} j}(t)=\phi\left(x_{i \pm \frac{1}{2}}, y_{j}, t\right) & \text { at midpoints of } y \text {-direction edges. }
\end{array}
$$

A VIA moment is defined over $\mathscr{C}_{i j}$ as

$$
\bar{V}_{i j}(t)=\frac{1}{\left|V_{i j}\right|} \int_{x_{i-\frac{1}{2}}}^{x_{i+\frac{1}{2}}} \int_{y_{i-\frac{1}{2}}}^{y_{i+\frac{1}{2}}} \phi(x, y, t) \mathrm{d} x \mathrm{~d} y,
$$

where $\left|V_{i j}\right|$ is the area of the control volume in two dimensions, for two-dimensional uniform grid in Cartesian coordinates $\left|V_{i j}\right|=\Delta x \times \Delta y$. As we will see later, the PV moments defined on the boundary edges of the control volume provide the sampled values for numerical quadrature of the desired accuracy for the computation of the flux during updating the VIA moment.

With the moments given above, a quadratic interpolation function can be constructed for control volume $\mathscr{C}_{i j}$ as follows:

$$
\Phi_{i j}(x, y)=c_{i j}^{00}+c_{i j}^{10} \tilde{x}+c_{i j}^{01} \tilde{y}+c_{i j}^{11} \tilde{x} \tilde{y}+c_{i j}^{20} \tilde{x}^{2}+c_{i j}^{02} \tilde{y}^{2}+c_{i j}^{21} \tilde{x}^{2} \tilde{y}++c_{i j}^{12} \tilde{x} \tilde{y}^{2}+c_{i j}^{22} \tilde{x}^{2} \tilde{y}^{2},
$$

where $\tilde{x}=x-x_{i-1 / 2}$ and $\tilde{y}=y-y_{j-1 / 2}$.

The nine coefficients are uniquely determined by nine constraint conditions in terms of the moments defined in (18) and (19). The detailed expressions can be referred to the appendix of [26].

The governing equation of conservative form for two-dimensional advection is

$$
\frac{\partial \phi}{\partial t}+\nabla \cdot(\mathbf{v} \phi)=0
$$

where $\phi$ is the transported field and $\mathbf{v}$ is the velocity vector.

To update the PV moment, the equation of advection form is adopted as

$$
\frac{\mathrm{D} \phi}{\mathrm{D} t}=-\phi \nabla \cdot \mathbf{v}
$$


Let $\bar{P}_{i^{\prime} j^{\prime}}$ denote any of the eight PV moments defined in (18), and $i^{\prime} j^{\prime}$ be the corresponding subscript index. Considering a divergence-free flow field, provided $\Phi^{n}(\mathbf{x})$,the spatial reconstruction of the physical field $\phi$ at time step $t^{n}$, we advance any PV moment $\bar{P}_{i^{\prime} j^{\prime}}$ defined on the edges of the control volume by using the Lagrangian invariant solution, as

$$
\bar{P}_{i^{\prime} j^{\prime}}^{n+1}=\Phi_{I J}^{n}\left(\mathbf{x}_{i^{\prime} j^{\prime}}^{P}\right)
$$

where $\Phi_{I J}^{n}$ is the interpolation function for $(I, J)$ cell at time $t^{n}, \mathbf{x}_{i^{\prime} j^{\prime}}^{P}$ is the departure point for point $\mathbf{x}_{i^{\prime} j^{\prime}}$ and $(I, J)$ is the cell index in which the departure point $\mathbf{x}_{i^{\prime} j^{\prime}}^{P}$ falls in.

For variable velocity field, the location of the departure point $\mathbf{x}_{i^{\prime} j^{\prime}}^{P}$ should be solved from the following IVP at $t=t^{n+1}$ :

$$
\begin{aligned}
& \frac{\mathrm{d} \mathbf{x}}{\mathrm{d} t}=-\mathbf{v}, \\
& \mathbf{x}\left(t=t^{n}\right)=\mathbf{x}_{i^{\prime} j^{\prime}} .
\end{aligned}
$$

A third-order Runge-Kutta scheme is applied to solve (24) as

$$
\mathbf{x}_{i^{\prime} j^{\prime}}^{P}=\mathbf{x}_{i^{\prime} j^{\prime}}-\frac{1}{4}\left(\mathbf{k}_{1}+3 \mathbf{k}_{3}\right)
$$

where

$$
\begin{aligned}
& \mathbf{k}_{1}=\mathbf{v}\left(\mathbf{x}_{i^{\prime} j^{\prime}}\right) \Delta t \\
& \mathbf{k}_{2}=\mathbf{v}\left(\mathbf{x}_{i^{\prime} j^{\prime}}-\frac{1}{3} \mathbf{k}_{1}\right) \Delta t, \\
& \mathbf{k}_{3}=\mathbf{v}\left(\mathbf{x}_{i^{\prime} j^{\prime}}-\frac{2}{3} \mathbf{k}_{2}\right) \Delta t .
\end{aligned}
$$

To control the non-physical oscillations, we again limit the PV moment by the local extremes. For two-dimensional control volume $\mathscr{C}_{i j}$, we set the local extremes as

$$
\begin{aligned}
& \phi_{i j}^{+}=\max \left(\phi_{i \pm \frac{1}{2} j \pm \frac{1}{2}}^{n}, \phi_{i \pm \frac{1}{2} j}^{n}, \phi_{i j \pm 1 / 2}^{n}\right), \\
& \phi_{i j}^{-}=\min \left(\phi_{i \pm \frac{1}{2} j \pm \frac{1}{2}}^{n}, \phi_{i \pm \frac{1}{2} j}^{n}, \phi_{i j \pm 1 / 2}^{n}\right) .
\end{aligned}
$$

Then the PV moments at $t^{n+1}$ is finally computed as

$$
\bar{P}_{i^{\prime} j^{\prime}}^{n+1}=\max \left\{\phi_{I J}^{-}, \min \left[\phi_{I J}^{+}, \Phi_{I J}^{n}\left(\mathbf{x}_{i^{\prime} j^{\prime}}^{P}\right)\right]\right\}
$$

Similar to one-dimensional case, by integrating (22), with the application of the Gauss theorem, a flux-form formulation for advancing the VIA is given as

$$
\frac{\partial^{V} \bar{\phi}_{i j}}{\partial t}=-\frac{1}{\left|V_{i j}\right|} \sum_{p=1}^{4} h_{p}=-\frac{1}{\left|V_{i j}\right|} \sum_{p=1}^{4} \int_{l_{p}} \mathbf{f} \cdot \mathbf{n}_{l_{p}} \mathrm{~d} l_{p},
$$

where $h$ is the line-integration of the numerical flux, $\mathbf{f}=\left(f^{x}, f^{y}\right)=\mathbf{v} \phi$ is the flux vector, $l$ denotes the edges encompassing the control volume and $\mathbf{n}$ is the outward unit normal vector of the boundary edges. For control volume $\mathscr{C}_{i j}$, we denote the boundary edges by $l_{1} \equiv \overline{P_{3} P_{4}}, l_{2} \equiv \overline{P_{4} P_{2}}, l_{3} \equiv \overline{P_{2} P_{1}}$ and $l_{4} \equiv \overline{P_{1} P_{3}}$ as shown in Figure 3 . 


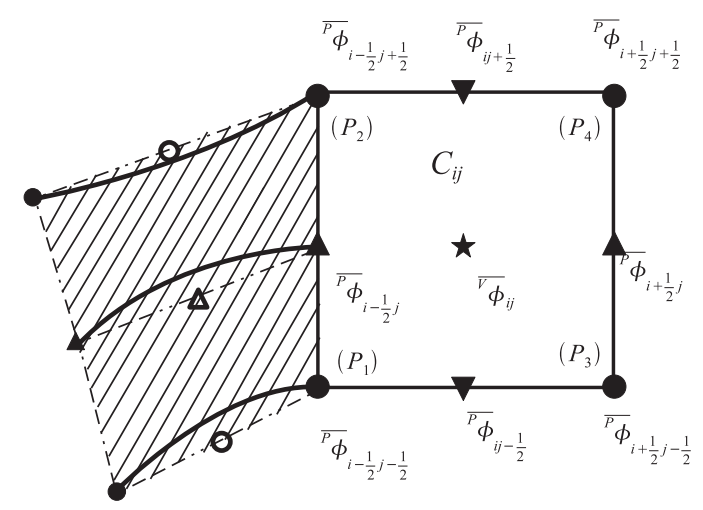

Figure 4. Updating procedure on two-dimensional plane.

The line-integration of the numerical flux is calculated by 3-point Simpson's rule (12) as

$$
\begin{aligned}
& h_{1}=\int_{l_{1}} \mathbf{f} \cdot \mathbf{n}_{l_{1}} \mathrm{~d} l=\frac{\Delta y}{6}\left(f_{i+\frac{1}{2} j-\frac{1}{2}}^{x}+4 f_{i+\frac{1}{2} j}^{x}+f_{i+\frac{1}{2} j+\frac{1}{2}}^{x}\right), \\
& h_{2}=\int_{l_{2}} \mathbf{f} \cdot \mathbf{n}_{l_{2}} \mathrm{~d} l=\frac{\Delta x}{6}\left(f_{i-\frac{1}{2} j+\frac{1}{2}}^{y}+4 f_{i j+\frac{1}{2}}^{y}+f_{i+\frac{1}{2} j+\frac{1}{2}}^{y}\right), \\
& h_{3}=\int_{l_{3}} \mathbf{f} \cdot \mathbf{n}_{l_{3}} \mathrm{~d} l=-\frac{\Delta y}{6}\left(f_{i-\frac{1}{2} j-\frac{1}{2}}^{x}+4 f_{i-\frac{1}{2} j}^{x}+f_{i-\frac{1}{2} j+\frac{1}{2}}^{x}\right), \\
& h_{4}=\int_{l_{4}} \mathbf{f} \cdot \mathbf{n}_{l_{4}} \mathrm{~d} l=-\frac{\Delta x}{6}\left(f_{i-\frac{1}{2} j-\frac{1}{2}}^{y}+4 f_{i j-\frac{1}{2}}^{y}+f_{i+\frac{1}{2} j-\frac{1}{2}}^{y}\right) .
\end{aligned}
$$

Concerning the solution of evolution equation (29), the time integration of $h$ is calculated in a way similar to (13) as

$$
g_{p}=\int_{t^{n}}^{t^{n+1}} h_{p} \mathrm{~d} t=\Delta t \sum_{q=1}^{M} \beta_{q} h_{p}\left(t^{n}+\alpha_{q} \Delta t\right) \quad(p=1 \text { to } 4) .
$$

The numerical fluxes needed in (30) are evaluated at the points where the PV moments are defined. To accomplish the computation, $\bar{P}_{i^{\prime} j^{\prime}}\left(t^{n}+\alpha_{q} \Delta t\right)$, the PV at $t^{n}+\alpha_{p} \Delta t$, is needed, which is calculated by the same semi-Lagrangian scheme as mentioned above to advance the PV moment with different time increments. As a more economical alternative, one can also simply specify the departure point at time of $t^{n}+\alpha_{q} \Delta t$ as $\left(1-\alpha_{q}\right) \mathbf{x}_{i^{\prime} j^{\prime}}+\alpha_{q} \mathbf{x}_{i^{\prime} j^{\prime}}^{P}$ instead of using the Runge-Kutta formulation (25) under the assumption that the trajectory is a straight line.

The VIA moment at time $t^{n+1}$ is then computed by

$$
\bar{V}_{i j}^{n+1}={\overline{{ }^{V}}}_{i j}^{n}-\frac{1}{\Delta x \Delta y} \sum_{p=1}^{4} g_{p}
$$

Figure 4 describes the numerical procedure to update PV moments and calculate the numerical flux at the left edge of control volume $\mathscr{C}_{i j}$. The moment configuration is same as Figure 3 . This procedure can be summarized as follows.

- Step 1. Departure points at $t=t^{n}$ are found for PV moments $\bar{P}_{i-(1 / 2) j}$ and $\bar{P}_{i-(1 / 2) j \pm 1 / 2}$ using the third-order Runge-Kutta scheme (25), which are shown by small solid makers in Figure 4 . The departure points at $t=t^{n}+\frac{1}{2} \Delta t$ (denoted by small hollow markers) are simply specified as the midpoints of straight lines connecting the arrival points and the departure points (dashed lines in Figure 4) for saving the computational costs.

- Step 2. Single-cell-based spatial reconstruction (20) is obtained based on known PVs and VIAs (at $t=t^{n}$ ) for each cell. 
- Step 3. Values at departure positions are computed through local spatial reconstruction polynomials. Each PV moment is updated by the Lagrangian invariant solution.

- Step 4. Numerical flux across the left edge of control volume $\mathscr{C}_{i j}$ (shadow area shown in Fig.4) is evaluated through nine points quadrature, including six departure points denoted by small markers and three arrival points along the left edge of control volume.

- Step 5. VIA moment is predicted by using flux-form formulation (32).

It should be noted that the present multi-moment scheme needs more computer storage for the extra moments. However, the computation can be carried out in a very efficient way. The twodimensional reconstruction is needed only for the semi-Lagrangian updating for the PV moment defined on the boundary of the control volume. The VIA moment is updated with the fluxes efficiently computed from quadratures based on the PVs. Furthermore, increasing the local DOFs allows a larger time step for stability compared with refining the grid and using a single-moment method with the same number of DOFs.

We have so far described a multi-moment finite volume transport scheme on the two-dimensional plane. Based on the multi-moment concept, we adopt two kinds of moments, i.e. PV and VIA, locally defined on each mesh cell, which then increases the local DOFs, and a quadratic spatial reconstruction can be built within a single mesh cell. With a compact stencil, the multi-moment scheme is well suited for the cubed-sphere grid, where the data transfer across the patch boundary can be carried out more accurately and efficiently.

\section{GLOBAL TRANSPORT MODEL ON CUBED SPHERE}

To extend the present scheme to the spherical geometry, we adopt the cubed-sphere grid proposed in [15]. This grid eliminates polar singularity in spherical LON/LAT grid and introduces a quasiuniform grid resolution for the whole globe. On a cubed sphere, the six identical patches covering the whole globe are separated by 12 patch boundaries which are created by projecting the edges of the inscribed cube as shown in Figure 5. The equiangular gnomonic projection is used in the present study to construct the local curvilinear coordinates on every patch; it results in a quasiuniform grid spacing and the analytic transformation laws for curvilinear coordinate systems. The procedure to construct the local coordinates on sphere by gnomonic projection is described in [21]. The coordinate system generated by gnomonic projection, however, is non-orthogonal, and the coordinates break along the patch boundaries. Thus, special treatment is needed to transfer data across the boundaries between different patches, which sometimes degrades the accuracy of the numerical models [20]. Numerical schemes that use compact grid stencil are more efficient and attractive because less halo zone for the data communication over the patch boundary is required. Based on the multi-moment concept, the spatial reconstruction of our method is accomplished within each single cell. This feature minimizes the data transfer across the patch boundary. In

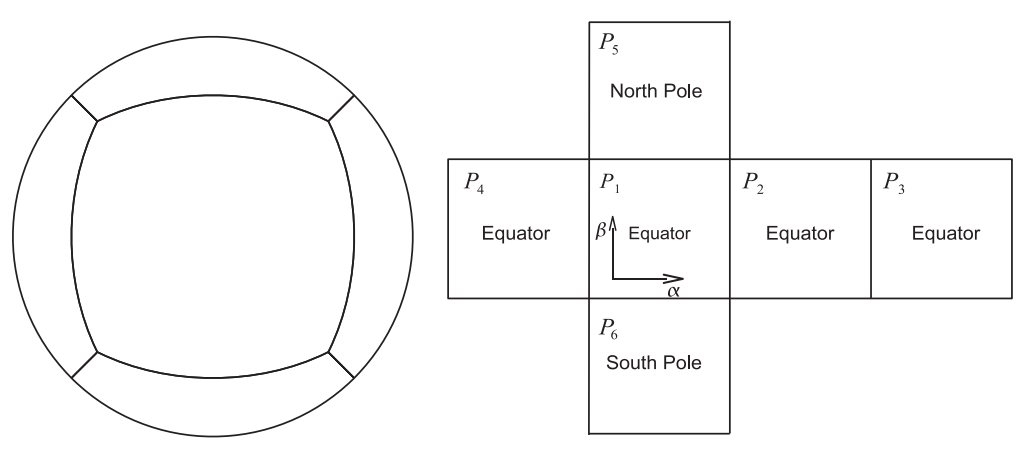

Figure 5. The cubed-sphere grid. 
our computation, the Lagrangian invariant solution can be directly evaluated from the single-cell interpolation where even the upstream point falls in another patch.

The flux-form governing equation for transport problem is now written in local curvilinear coordinates as [15]

$$
\frac{\partial \psi}{\partial t}+\frac{\partial(\tilde{u} \psi)}{\partial \xi}+\frac{\partial(\tilde{v} \psi)}{\partial \eta}=0
$$

where $(\xi, \eta)$ is the local curvilinear coordinate system, $\xi=R \alpha$ and $\eta=R \beta, R$ is the radius of the sphere, $\alpha$ and $\beta$ are central angles. $\psi=\sqrt{G} \phi$ and $\phi$ is the transported field. $(\tilde{u}, \tilde{v})$ is the contravariant velocity vector, and $\sqrt{G}$ the Jacobian of the transformation,

$$
\sqrt{G}=\left(1+\tan ^{2} \alpha+\tan ^{2} \beta\right)^{-3 / 2} \cos ^{-2} \alpha \cos ^{-2} \beta .
$$

On local curvilinear coordinates, the scheme developed for two-dimensional Cartesian grid in section 3 can be extended to spherical geometry straightforwardly.

The definition of PV and VIA moments is similar to (18) and (19). Considering control volume $\mathscr{C}_{i j k}$ where the third subscript denotes the index of the patch $(k=1$ to 6$)$, the location of the moments can be represented using Figure 3 through replacing coordinate system $(x, y)$ by $(\xi, \eta)$. Then, the PV moments of variable $\psi(\xi, \eta, t)$ are

$$
\begin{array}{ll}
\bar{P}_{i \pm \frac{1}{2} j \pm \frac{1}{2} k}(t)=\psi\left(\xi_{i \pm \frac{1}{2}}, \eta_{j \pm \frac{1}{2}}, k, t\right) & \text { at vertices, } \\
\bar{P}_{i j \pm 1 / 2 k}(t)=\psi\left(\xi_{i}, \eta_{j \pm \frac{1}{2}}, k, t\right) & \text { at midpoints of } \xi \text {-direction edges, } \\
\bar{P}_{i \pm \frac{1}{2} j k}(t)=\psi\left(\xi_{i \pm \frac{1}{2}}, \eta_{j}, k, t\right) & \text { at midpoints of } \eta \text {-direction edges, }
\end{array}
$$

and the VIA moment is

$$
\bar{V}_{i j k}(t)=\frac{1}{\left|V_{i j k}\right|} \int_{\xi_{i-\frac{1}{2}}}^{\xi_{i+\frac{1}{2}}} \int_{\eta_{i-\frac{1}{2}}}^{\eta_{i+\frac{1}{2}}} \psi(\xi, \eta, k, t) \mathrm{d} \xi \mathrm{d} \eta,
$$

where $\left|V_{i j k}\right|=\Delta \xi \times \Delta \eta$ is the area in computational space. Since the mass $m_{i j k}$ of transported field $\phi$ within element $\mathscr{C}_{i j k}$ is computed as

$$
m_{i j k}=\int_{\xi_{i-\frac{1}{2}}}^{\xi_{i+\frac{1}{2}}} \int_{\eta_{i-\frac{1}{2}}}^{\eta_{i+\frac{1}{2}}} \sqrt{G} \phi(\xi, \eta, k) \mathrm{d} \xi \mathrm{d} \eta,
$$

the VIA moment of model variable $\psi(\xi, \eta, t)$ is a quantity that should be conserved as the mass of the transported field $\phi(\xi, \eta, t)$.

The single-cell-based piecewise quadratic interpolation function $\Psi_{i j k}^{n}(\xi, \eta, k)$ can be constructed using (20) for variable $\psi$. As mentioned at the beginning of this section, a single-cell-based reconstruction profile does not require the ghost cells from the neighboring patches for boundary elements; it simplifies the numerical treatment of patch boundaries.

Considering a divergence-free flow field, the advection-form governing equation for updating the PV moment is

$$
\frac{\mathrm{D} \phi}{\mathrm{D} t}=0
$$

With the known interpolatants $\Psi^{n}(\chi)$ at time step $t^{n}$, the PV moment at the next time step can be updated by the Lagrangian invariant solution. If the departure point of grid point $\xi_{i^{\prime} j^{\prime} k}$ is $\xi_{i^{\prime} j^{\prime} k}^{P}$, the PV moment at the next time step is obtained as

$$
\bar{P}_{i^{\prime} j^{\prime} k}^{n+1}=\frac{\bar{G}_{i^{\prime} j^{\prime} k}}{\sqrt{G}_{i^{\prime} j^{\prime} k}} \Psi_{I J K}^{n}\left(\xi_{i^{\prime} j^{\prime} k}^{P}\right),
$$


where subscripts $I, J, K$ denote the element which the departure point falls in, $\sqrt{G}_{i^{\prime} j^{\prime} k}$ is the Jacobian of the transformation at arrival point and $\sqrt{G}_{i^{\prime} j^{\prime} k}^{P}$ is Jacobian at the departure point.

In present study, the trajectory calculation is carried out in three-dimensional Cartesian coordinates $(x, y, z)$ [31]. The Runge-Kutta scheme is also adopted as in Section 3 but in three dimensions. Before calculation, we project the location of the arrival point to Cartesian grid using $x=R \cos \theta \cos \lambda, y=R \cos \theta \sin \lambda$ and $z=R \sin \theta$. Constrained on the sphere surface, the trajectory must satisfy the $\left|\mathbf{x}^{P}\right|=R$ [32]. Hence, the trajectory after the Runge-Kutta computation should be further corrected as $\mathbf{x}^{P}=R \mathbf{x}^{P} /\left|\mathbf{x}^{P}\right|$. Then the location of the departure point $\mathbf{x}^{P}=\left(x^{P}, y^{P}, z^{P}\right)$ are re-projected back to the local grid. The departure point is represented by $(\xi, \eta, I, J, K)$ in our implementation, which means that the departure point is located within $(I, J)$ element of $K$ th patch, and the local coordinates are $(\xi, \eta)$. It should be pointed out that the task to pass the information between the different patches is embedded in this procedure for the present scheme.

After advancing the PV moment, the VIA moment $\bar{V}^{V}{ }_{i j k}$ is updated by integrating the governing Equation (33),

$$
\frac{\partial^{V} \bar{\psi}_{i j k}}{\partial t}=-\frac{1}{\left|V_{i j k}\right|} \sum_{p=1}^{4} \int_{l_{p}} \mathbf{f} \cdot \mathbf{n}_{l_{p}} \mathrm{~d} l_{p},
$$

where $\mathbf{f}=(\tilde{u} \psi, \tilde{v} \psi)$ is the flux vector; the other symbols are the same as in (29) but in a mapped Cartesian mesh element in the computational space.

Owing to the similarity between (29) and (40), the remaining work is implemented using similar formulations developed in Section 3 for transport problem on the two-dimensional plane. The numerical fluxes across the patch boundaries are computed by the same PVs on the different patches; the numerical conservation of the VIA moment guarantees the mass conservation over the whole globe.

\section{NUMERICAL TESTS}

To validate our numerical model, several numerical tests have been carried out in this section. To quantify the performance of the scheme, we check the normalized errors, which are defined following [29]. The numerical errors are evaluated in terms of the VIA moment, which is the conserved primary variable in the numerical model.

\subsection{Convergence test}

We use numerical tests to verify the third-order accuracy of the present scheme on the cubed grid. The numerical experiments are carried out on a series of refined grids without using limiter.

The initial distribution is given in rotated LON/LAT coordinates $\left(\lambda^{\prime}, \theta^{\prime}\right)$ as

$$
\phi\left(\lambda^{\prime}, \theta^{\prime}, 0\right)=\cos ^{p} \theta^{\prime} \sin k \lambda^{\prime},
$$

where the origin of the rotated grid is located at $(0, \alpha)$ of the original grid, the transformation is computed by

$$
\begin{aligned}
& \lambda^{\prime}=\arctan \left(\frac{\cos \theta \sin \lambda}{\cos \theta \cos \lambda \cos \alpha+\sin \theta \sin \alpha}\right), \\
& \theta^{\prime}=\arcsin (\sin \theta \cos \alpha-\cos \theta \cos \lambda \sin \alpha) .
\end{aligned}
$$

A divergence-free velocity field is specified as [29]

$$
\begin{aligned}
& u_{\lambda}=u_{0}(\cos \theta \cos \alpha+\sin \theta \cos \lambda \sin \alpha), \\
& u_{\theta}=-u_{0} \sin \lambda \sin \alpha,
\end{aligned}
$$

where $u_{0}=2 \pi R /(12$ days $)$. 
Table II. Convergence test on cubed-sphere grid.

\begin{tabular}{|c|c|c|c|c|c|c|}
\hline \multirow[b]{2}{*}{ Grid } & \multicolumn{2}{|c|}{$l_{1}$} & \multicolumn{2}{|c|}{$l_{2}$} & \multicolumn{2}{|c|}{$l_{\infty}$} \\
\hline & Error & Order & Error & Order & Error & Order \\
\hline $16 \times 16 \times 6$ & $9.9375 e-3$ & - & $9.7149 \mathrm{e}-3$ & - & $1.0380 \mathrm{e}-2$ & - \\
\hline $32 \times 32 \times 6$ & $1.2944 \mathrm{e}-3$ & 2.94 & $1.2522 \mathrm{e}-3$ & 2.96 & $1.3757 \mathrm{e}-3$ & 2.92 \\
\hline $64 \times 64 \times 6$ & $1.7052 \mathrm{e}-4$ & 2.92 & $1.6203 \mathrm{e}-4$ & 2.95 & $1.8397 \mathrm{e}-4$ & 2.90 \\
\hline
\end{tabular}

Table III. The normalized errors of cosine bell advection after one revolution (256 steps) on the grid of $32 \times 32 \times 6$ with limiting projection for suppressing numerical oscillation.

\begin{tabular}{lccccr}
\hline & $l_{1}$ error & $l_{2}$ error & $l_{\infty}$ error & MAX error & MIN error \\
\hline$\alpha=0$ & $3.7247 \mathrm{e}-2$ & $2.4904 \mathrm{e}-2$ & $2.4832 \mathrm{e}-2$ & $-1.4019 \mathrm{e}-2$ & $-1.7652 \mathrm{e}-3$ \\
$\alpha=\frac{\pi}{4}$ & $3.2355 \mathrm{e}-2$ & $2.2508 \mathrm{e}-2$ & $2.7915 \mathrm{e}-2$ & $-1.4168 \mathrm{e}-2$ & $-1.1310 \mathrm{e}-3$ \\
$\alpha=\frac{\pi}{2}$ & $3.7368 \mathrm{e}-2$ & $2.5211 \mathrm{e}-2$ & $2.4837 \mathrm{e}-2$ & $-1.4001 \mathrm{e}-2$ & $-1.7709 \mathrm{e}-3$ \\
\hline
\end{tabular}

Table IV. Same as Table III, but without limiting projection.

\begin{tabular}{lccccr}
\hline & $l_{1}$ error & $l_{2}$ error & $l_{\infty}$ error & MAX error & MIN error \\
\hline$\alpha=0$ & $4.6122 \mathrm{e}-2$ & $2.9640 \mathrm{e}-2$ & $2.4012 \mathrm{e}-2$ & $-1.3984 \mathrm{e}-2$ & $-1.2796 \mathrm{e}-2$ \\
$\alpha=\frac{\pi}{4}$ & $4.3362 \mathrm{e}-2$ & $2.6227 \mathrm{e}-2$ & $1.6324 \mathrm{e}-2$ & $-1.5872 \mathrm{e}-2$ & $-7.3005 \mathrm{e}-3$ \\
$\alpha=\frac{\pi}{2}$ & $4.6337 \mathrm{e}-2$ & $2.9786 \mathrm{e}-2$ & $2.4031 \mathrm{e}-2$ & $-1.3965 \mathrm{e}-2$ & $-1.2796 \mathrm{e}-2$ \\
\hline
\end{tabular}

The flow field specified by (43) represents a solid rotation; the parameter $\alpha$ is the angle between the rotation axis and the polar axis of the Earth.

In this test, we specify $\alpha=\pi / 4, p=4$ and $k=4$. The CFL number is 0.25 . On the cubed-sphere grid, the case of $\alpha=\pi / 4$ is of much interest. In this case, the flow trajectory passes two complete boundaries and four vertices of the inscribed cube. The results of the tests on the grids with different resolutions are shown in Table II. The third-order accuracy is verified.

\subsection{Cosine bell advection}

This benchmark test is proposed in [29] to examine the global advection transport on sphere. The initial distribution is given as

$$
\phi(\lambda, \theta, 0)= \begin{cases}\left(\frac{h_{0}}{2}\right)\left[1+\cos \left(\frac{\pi r}{r_{0}}\right)\right] & \text { if } r<r_{0}, \\ 0 & \text { otherwise }\end{cases}
$$

where $r$ is the great circle distance between the point $(\lambda, \theta)$ and the initial center $(3 \pi / 2,0)$, other constants are specified as $h_{0}=1000 \mathrm{~m}$ and $r_{0}=(7 \pi / 64) R$.

The divergence-free flow field is given by (43).

The numerical experiment is carried out on a $32 \times 32 \times 6$ grid with three rotation directions of $\alpha=0, \alpha=\pi / 4$ and $\alpha=\pi / 2$. The CFL number is specified as 0.5 here for comparing the results with other existing schemes. The normalized errors of the numerical results with and without the limiter are given in Tables III and IV, respectively. The numerical results for this test by several existing schemes on spherical geometry are listed in Table III of [33]. It is revealed that our scheme is competitive to those methods. In particular, completely getting around the polar problem in the direction of $\alpha=\pi / 2$, our scheme is apparently superior to those methods. The cosine bell advection is also tested in $[12,16,17,22]$ by other existing schemes on the cubic grid. We found that the present scheme gives better result compared with the semi-Lagrangian schemes 

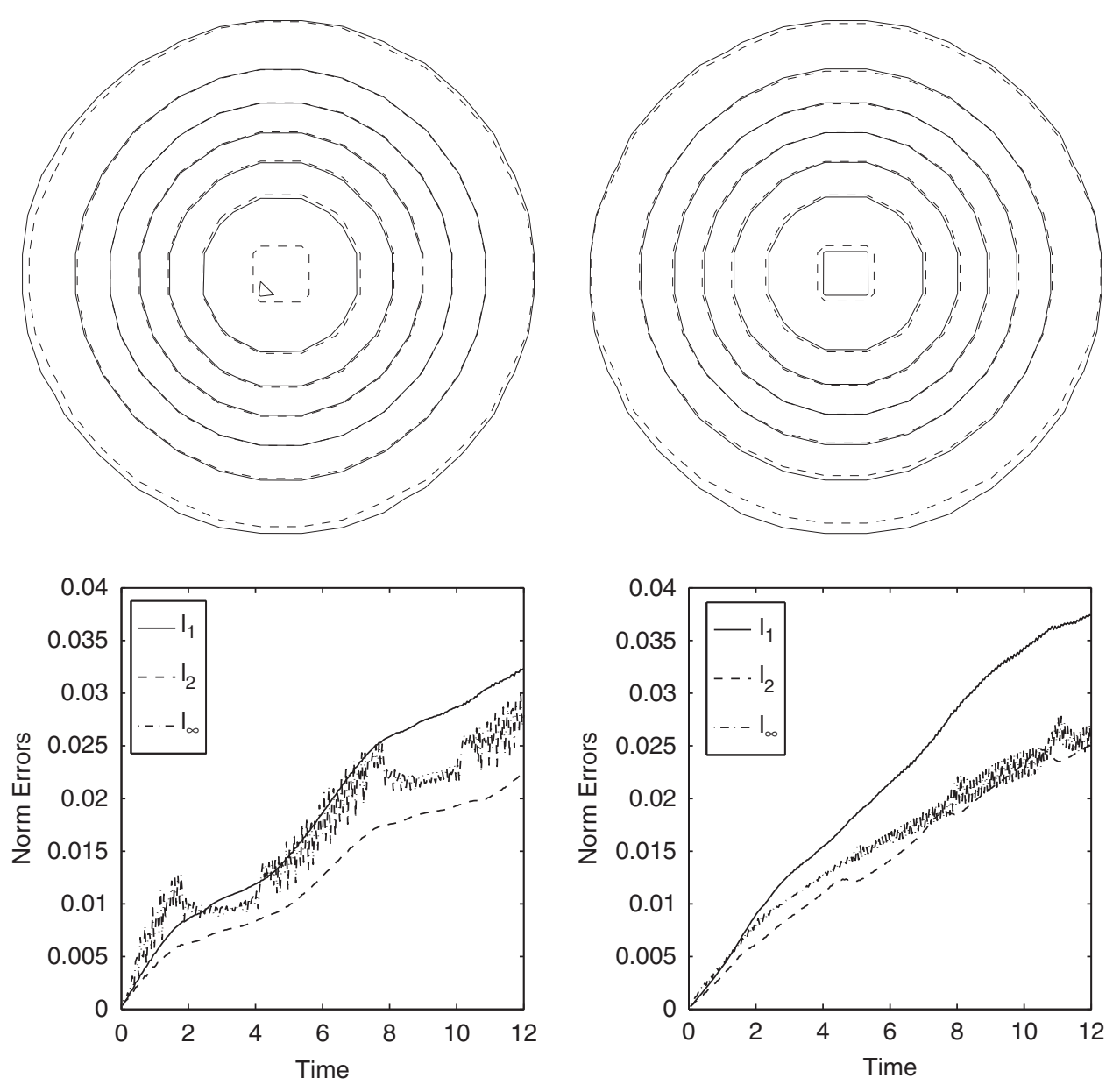

Figure 6. The results and normalized errors of cosine bell advection test. The contour plots are the results after 12 days in the directions of $\alpha=\pi / 4$ (top-left) and $\alpha=\pi / 2$ (top-right), contour lines vary from 50 to $950 \mathrm{~m}$ by $150 \mathrm{~m}$, solid lines denote numerical result and dashed lines denote the initial distribution. The evolution of the normalized errors within one revolution is displayed on the bottom-left panel for $\alpha=\pi / 4$ and bottom-right panel for $\alpha=\pi / 2$.

developed on both the gnomonic [17] and the conformal [16] cubic grids with the same CFL number and similar grid resolution. According to the normalized errors, it is also observed that our scheme performs better than the TVD scheme reported in [22] and the conservative semiLagrangian scheme CSLAM proposed in [12]. As mentioned above, special attention should be paid to the flow in the direction of $\alpha=\pi / 4$, a case where most cubed-sphere grid-based models produce significant numerical errors. From our results, the flow in this direction does not generate larger errors compared with other directions. Figure 6 displays the contour plots of numerical results and the evolution of the normalized errors in the directions of $\alpha=\pi / 4$ and $\alpha=\pi / 2$. There is no obvious oscillations in the error plots when cosine bell moves across or along the patch boundaries.

We also ran this test with CFL number of 0.8. The normalized errors for flow in the direction of $\alpha=\pi / 4$ is given in Table V. The numerical result shows that the present scheme performs better with a larger CFL number. To check the performance of trajectory calculation method adopted in the present scheme, this test case is also carried out with exact trajectory and the normalized errors are given in Table VI. Errors shown in Tables V and VI are very close, which reveals that the method adopted here can compute the trajectory with adequate accuracy. 
Table V. Same as Table III, but with larger CFL number (160 steps for one revolution) and in the direction of $\alpha=\pi / 4$.

\begin{tabular}{cccccc}
\hline & $l_{1}$ error & $l_{2}$ error & $l_{\infty}$ error & MAX error & MIN error \\
\hline$\alpha=\frac{\pi}{4}$ & $1.9707 \mathrm{e}-2$ & $1.4433 \mathrm{e}-2$ & $1.8441 \mathrm{e}-2$ & $-8.2610 \mathrm{e}-3$ & $-1.3980 \mathrm{e}-3$ \\
\hline
\end{tabular}

Table VI. Same as Table V, but with exact trajectory.

\begin{tabular}{cccccc}
\hline & $l_{1}$ error & $l_{2}$ error & $l_{\infty}$ error & MAX error & MIN error \\
\hline$\alpha=\frac{\pi}{4}$ & $1.9426 \mathrm{e}-2$ & $1.3539 \mathrm{e}-2$ & $1.8525 \mathrm{e}-2$ & $-8.2467 \mathrm{e}-3$ & $-1.3065 \mathrm{e}-3$ \\
\hline
\end{tabular}
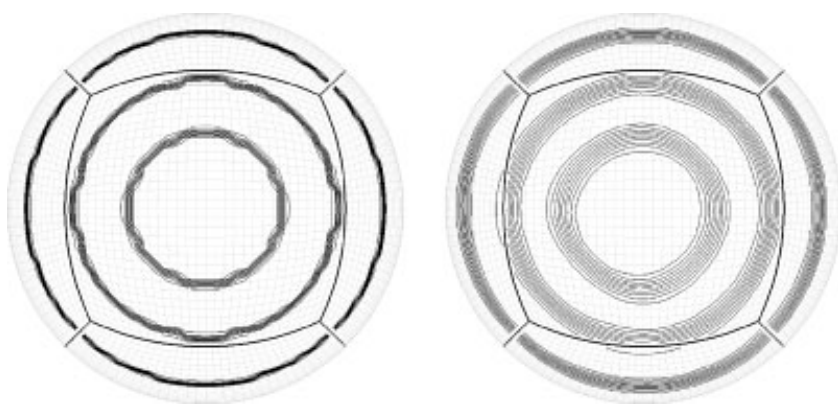

Figure 7. The result of the advection of the discontinuities, exact solution (left), numerical result (right) after integrating for 60 days on $32 \times 32 \times 6$ grid with 800 steps, contour lines vary from 0.1 to 0.9 by 0.1 .

\subsection{Advection of the discontinuities}

A test where the transported profile is discontinuous was used in [22]; the initial distribution is given as

$$
\phi(\lambda, \theta)= \begin{cases}1 & \text { if } r<0.4 R \\ 1 & \text { if } 0.72 R<r<1.12 R \\ 0 & \text { otherwise }\end{cases}
$$

where $r$ is the great circle distance from the center located at $(3 \pi / 2,0)$. The flow field is a rotation field specified by (43) with $\alpha=\pi / 4$ here.

After integrating the model for five revolutions ( 60 days) on $32 \times 32 \times 6$ grid with 800 time steps $(\mathrm{CFL}=0.8)$, the contour plots of the exact solution and the numerical result with limiting projection are given in Figure 7. The shape of the discontinuities are preserved very well. The bird's eye views of the results on patch four from the computations with and without the numerical limiter are shown in Figure 8. The normalized errors are $l_{1}=1.9586 \times 10^{-1}, l_{2}=2.3814 \times 10^{-1}, l_{\infty}=5.6762 \times 10^{-1}$, $\mathrm{MAX}=1.7933 \times 10^{-3}, \mathrm{MIN}=-3.4993 \times 10^{-3}$ for result with the limiter and $l_{1}=2.2596 \times 10^{-1}$, $l_{2}=2.1756 \times 10^{-1}, l_{\infty}=3.9004 \times 10^{-1}, \operatorname{MAX}=9.8542 \times 10^{-2}, \mathrm{MIN}=-1.2548 \times 10^{-1}$ for that without the limiter. With the application of the PV limiter, the spurious oscillations are completely eliminated for the PV moment and effectively suppressed for the VIA moment.

\subsection{Deformational flow on the sphere}

We evaluated the present scheme with a more challenging deformational flow test introduced in $[11,34]$. By adjusting the parameters in the initial distribution, both smooth and non-smooth profiles can be obtained. We tested both cases, and used the limiting projection in the non-smooth case. 

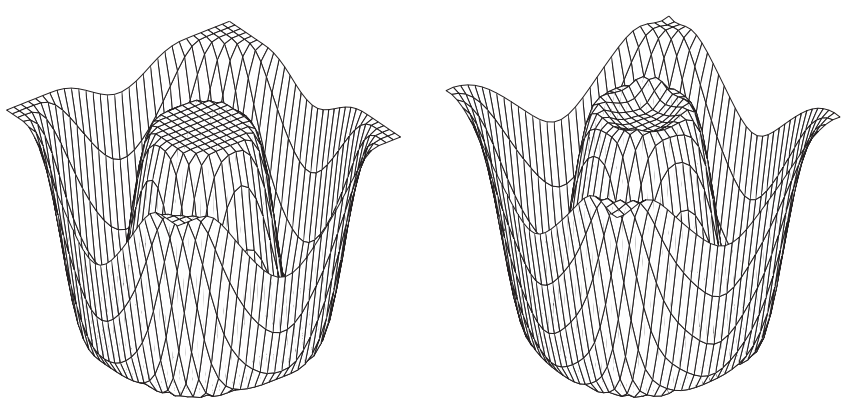

Figure 8. Bird's eye views of the solution on Patch IV. Displayed are the numerical results with (left) and without (right) the limiting projection.

The smooth case. In a rotated LON/LAT coordinate $\left(\lambda^{\prime}, \theta^{\prime}\right)$ on a unit sphere, the tangential velocity is specified as

$$
u_{\lambda}^{\prime}=\frac{3 \sqrt{3}}{2} \operatorname{sech}^{2}(\rho) \tanh (\rho),
$$

where $\rho=\gamma \cos \theta^{\prime}$ and the angular velocity is $\omega^{\prime}=u_{\lambda}^{\prime} / \rho$.

The initial transported field is specified as

$$
\phi\left(\lambda^{\prime}, \theta^{\prime}, 0\right)=1-\tanh \left(\frac{\rho}{\delta} \sin \lambda^{\prime}\right),
$$

and the analytic solution is given as

$$
\phi\left(\lambda^{\prime}, \theta^{\prime}, t\right)=1-\tanh \left[\frac{\rho}{\delta} \sin \left(\lambda^{\prime}-\omega^{\prime} t\right)\right] .
$$

We set $\gamma=3$ and $\delta=5$ in the smooth test [11]. The initial height field is displayed in the top-left panel of Figure 9. We put the origin of the rotated LON/LAT grid at $(-\pi / 4,-3 \pi / 10)$. The centers of two vortexes defined by flow field are located at the two vertices of the inscribed cube. It is quite challenging for the cubed-sphere grid. We ran the computation on a $32 \times 32 \times 6$ grid for 64 steps to $t=3$ without limiting. The numerical results are given in the bottom-left panel in Figure 9 with the exact solution in the top-right panel for comparison. The normalized errors are $l_{1}=6.5158 \times 10^{-5}$, $l_{2}=2.3880 \times 10^{-4}, l_{\infty}=1.4684 \times 10^{-3}$. There is nearly no visible difference between our result and the analytic one; the absolute error of the transported field is shown in the bottom-right panel in Figure 9.

5.4.1. The non-smooth test. The flow field is defined by (46), but with the parameters specified as

$$
\rho=\frac{2 \gamma \cos \theta^{\prime}}{1+\sin \theta^{\prime}} \quad \text { and } \quad \omega^{\prime}=\frac{u_{\lambda}^{\prime}}{\cos \theta^{\prime}} .
$$

The advected field is given at initial time as

$$
\phi\left(\lambda^{\prime}, \theta^{\prime}, 0\right)=-\tanh \left(\frac{\rho}{\gamma \delta} \sin \lambda^{\prime}\right),
$$

and the analytic solution is

$$
\phi\left(\lambda^{\prime}, \theta^{\prime}, t\right)=-\tanh \left[\frac{\rho}{\gamma \delta} \sin \left(\lambda^{\prime}-\omega^{\prime} t\right)\right] .
$$

The parameters of $\gamma=1.5$ and $\delta=0.02$ are set to obtain a non-smooth initial profile. The test is carried out on a $32 \times 32 \times 6$ grid with the PV limiter. The numerical results after 2.5 time units with 64 steps is shown in the left panel of Figure 10. Compared with the analytic solution shown 

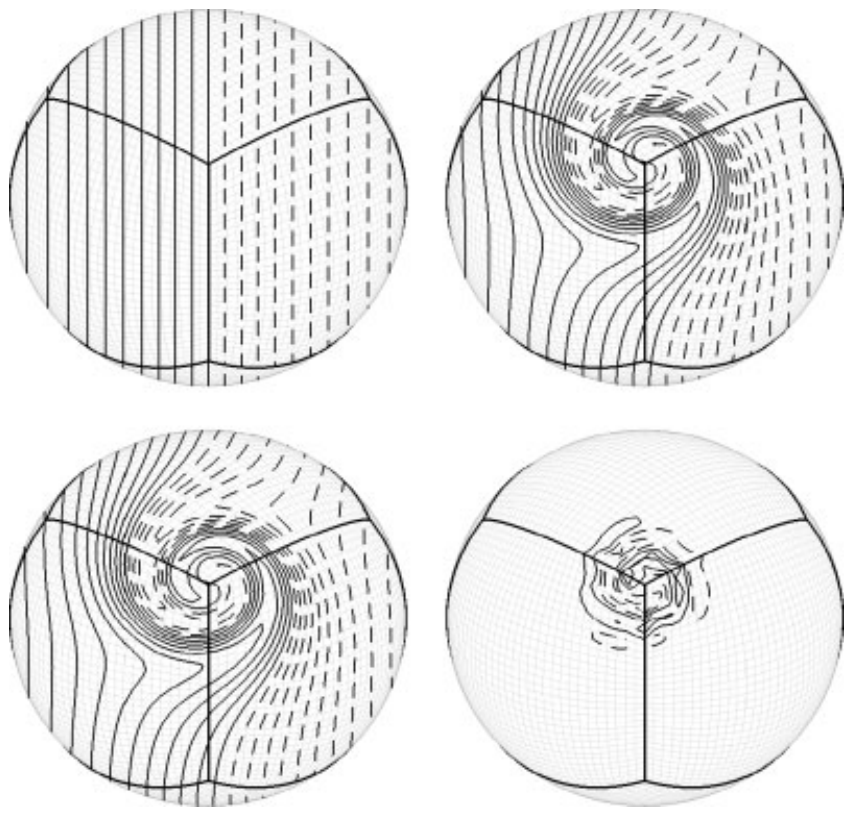

Figure 9. The numerical results of the smooth deformational flow. Shown are initial distribution (top-left), exact solution (top-right), numerical result (bottom-left) and absolute error (bottom-right). Contour lines vary in $[0.5,1.5]$ by 0.05 for height field with dashed lines for transport field lower than 1 and solid lines for transport field higher than 1 . In error plot, the dashed lines vary from $-2.5 \times 10^{-3}$ to $-5 \times 10^{-4}$ and solid lines vary from $5 \times 10^{-4}$ to $2.5 \times 10^{-3}$ by $1 \times 10^{-3}$.
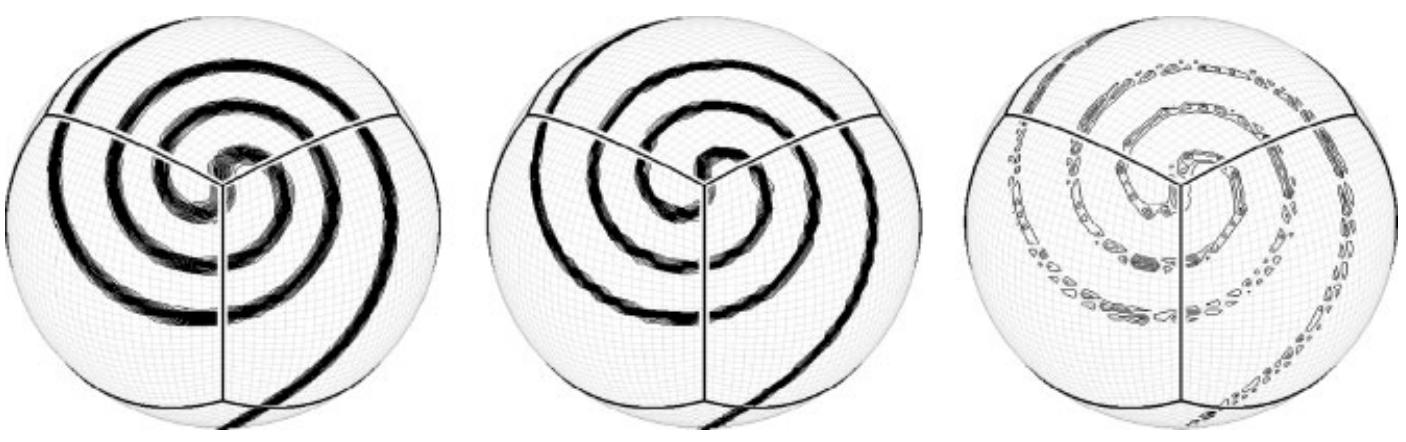

Figure 10. The result of the deformational flow with a non-smooth initial profile. Displayed are the numerical results after 2.5 time units (left) and the analytic solution (middle). Contour lines vary from -0.9 to 0.9 by 0.1 . Absolute error is shown in right panel with contour lines varying from -0.65 to 0.65 by 0.13 .

in the middle panel of Figure 10, we find that the steepness of the initial profile is adequately preserved, and the phase of the moving front is accurately reproduced. The normalized errors for this test are $l_{1}=1.9287 \times 10^{-2}, l_{2}=6.7869 \times 10^{-2}, l_{\infty}=6.5156 \times 10^{-1}$ and MAX $=3.0547 \times 10^{-2}$, $\mathrm{MIN}=-2.3186 \times 10^{-2}$.

\subsection{Moving vortices on the sphere}

A new test with moving vortices was recently proposed in [35]. Different from the previous test case where the centers of vortices are stationary, the vortex centers travel at the velocity of the solid rotation. To specify the flow field, the location of vortex center is determined at first, then the flow field is specified as the summation of the deformational and solid-rotational motions. The details of this test can be found in [35]. In this test, we define the initial condition with the vortex 

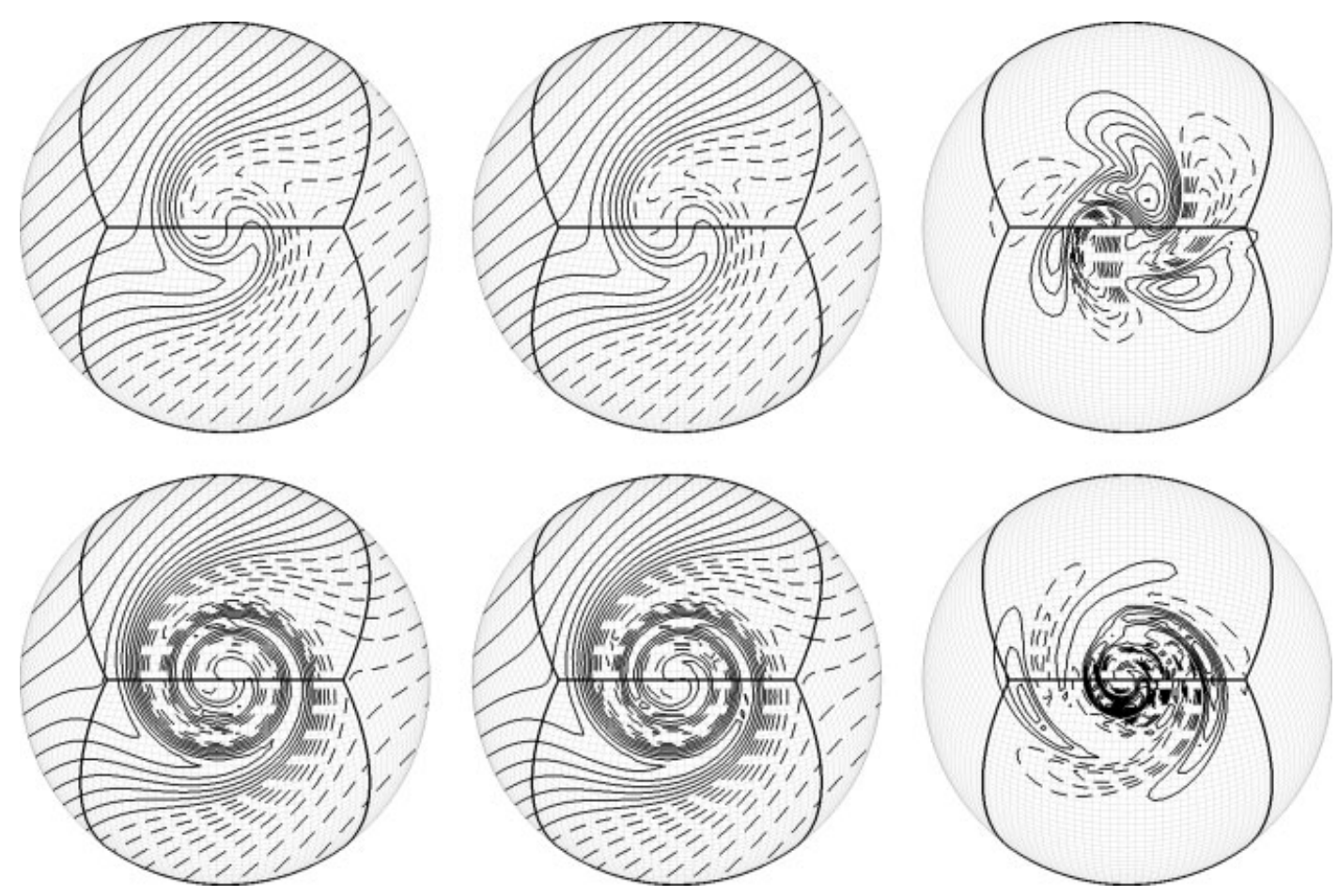

Figure 11. Advected field for moving vortices case with $\alpha=\pi / 4$. Shown are numerical result (top-left) and exact solution(top-middle) at day 3, numerical result (bottom-left) and exact solution (bottom-middle) at day 9. Contour lines are distributed same as in Figure 9. Absolute errors are illustrated in top-right panel for result at day 3 with contour lines varying from -0.02 to 0.02 by 0.002 and bottom-right panel for result at day 9 with contour lines varying from -0.05 to 0.05 by 0.002 . Negative lines are dashed and positive lines are solid.

center initially located at $(3 \pi / 2,0)$. On the cubed-sphere grid, two rotation directions are selected for vortex center, i.e. the parameter $\alpha$ in (43) is chosen as $\alpha=0$ and $\alpha=\pi / 4$, respectively.

Numerical experiments were carried out on three gradually refined grids of $18 \times 18 \times 6,36 \times$ $36 \times 6$ and $72 \times 72 \times 6$ with steps of 144,288 , and 576 , respectively. The numerical results on $36 \times 36 \times 6$ grid together with exact solutions and absolute errors are shown in Figure 11 for test with $\alpha=\pi / 4$. Proposed scheme accurately reproduces the evolution of the transported fields. The normalized errors at day 12 are given in Table VII and time history of normalized errors is illustrated in Figure 12. Compared with the results in [35], the multi-moment model on $36 \times 36 \times 6$ grid is competitive to the finite volume method with the same DOFs, i.e. $1.25^{\circ} \times 1.25^{\circ}$ uniform grid, and better than the semi-Lagrangian model with the same resolution. On $72 \times 72 \times 6$ grid, our result is competitive to that by the CSLAM scheme [12] with the similar resolution $(80 \times 80 \times 6)$. Compared with the DG solution, smaller oscillation is found in time history of normalized errors of $\alpha=\pi / 4$ case as shown in Figure 12. Furthermore, different from the semi-Lagrangian model in [35], we did not use the analytical upstream point in our calculations. The results again verify the accuracy of the trajectory calculation in present scheme.

\section{CONCLUSION}

A semi-Lagrangian multi-moment transport scheme is developed and extended to the spherical geometry through the cubed-sphere grid in this paper. The cubed-sphere grid results in a quasiuniform grid over the whole globe and is free of the polar problems. Based on the multi-moment concept, a single-cell reconstruction profile is adopted in this study, which essentially benefits the 
Table VII. Normalized errors of moving vortices case at day 12.

\begin{tabular}{lccccccc}
\hline & \multicolumn{3}{c}{$\alpha=0$} & & \multicolumn{3}{c}{$\alpha=\frac{\pi}{4}$} \\
\cline { 2 - 4 } \cline { 7 - 8 } Grid & $l_{1}$ & $l_{2}$ & $l_{\infty}$ & & $l_{1}$ & $l_{2}$ & $l_{\infty}$ \\
\hline $18 \times 18 \times 6$ & $8.7769 \mathrm{e}-3$ & $1.9338 \mathrm{e}-2$ & $7.0897 \mathrm{e}-2$ & & $7.3638 \mathrm{e}-3$ & $1.8342 \mathrm{e}-2$ & $7.4970 \mathrm{e}-2$ \\
$36 \times 36 \times 6$ & $3.9833 \mathrm{e}-3$ & $9.5294 \mathrm{e}-3$ & $4.6916 \mathrm{e}-2$ & & $3.4513 \mathrm{e}-3$ & $9.5012 \mathrm{e}-3$ & $5.2330 \mathrm{e}-2$ \\
$72 \times 72 \times 6$ & $1.3764 \mathrm{e}-3$ & $2.7992 \mathrm{e}-3$ & $1.5773 \mathrm{e}-2$ & & $1.2535 \mathrm{e}-3$ & $3.1687 \mathrm{e}-3$ & $1.8959 \mathrm{e}-2$ \\
\hline
\end{tabular}
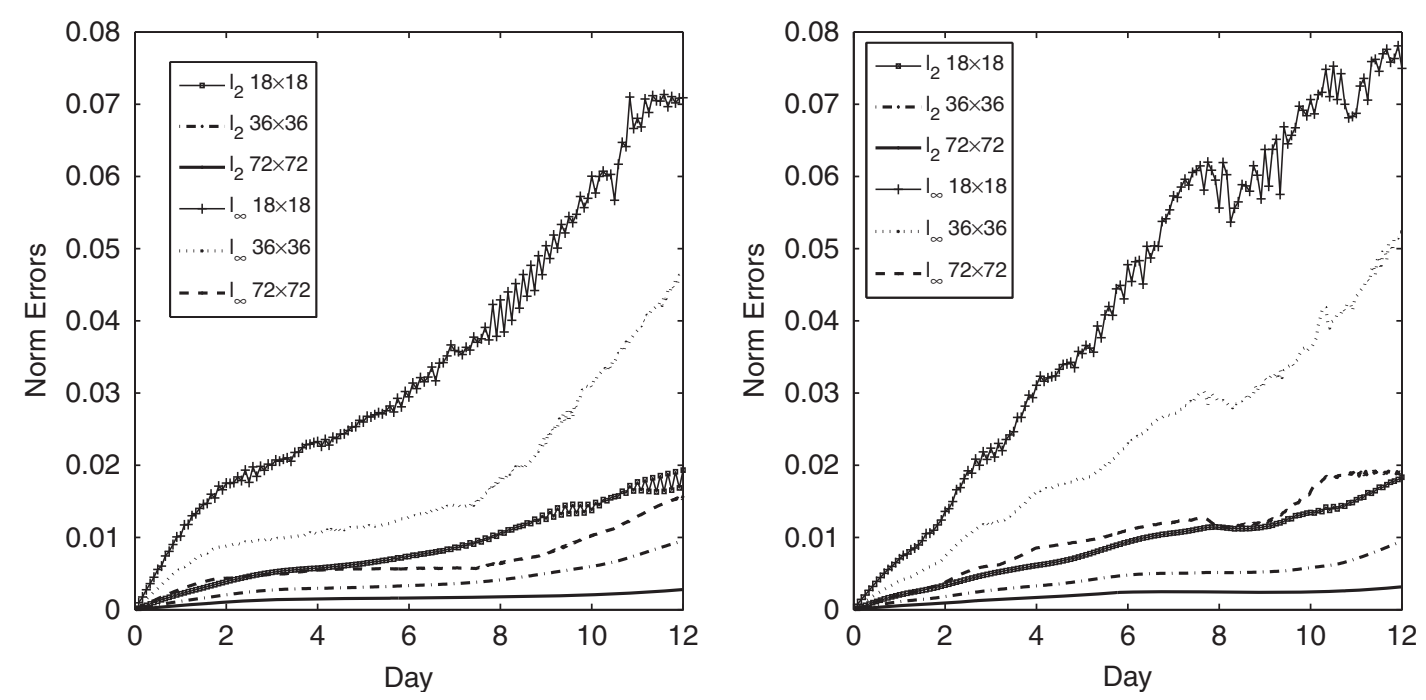

Figure 12. Time history of normalized errors for moving vortices case with $\alpha=0$ (left) and $\alpha=\pi / 4$ (right).

numerical treatment over the patch boundaries of the cubed-sphere grid. The resulting model is more robust in advection computation in different flow directions compared with other existing schemes. In terms of the VIA moment, the proposed transport model exactly guarantees the conservation over the whole globe. For practical use, we introduced a simple monotonic limiting projection to the PV moment in the presence of discontinuities, which completely removes the spurious oscillations from the PV moment and effectively suppresses those in the VIA moment. The benchmark tests verified the features of the present method, which can be a practical numerical tool for advection transport in spherical geometry.

\section{APPENDIX A: ANALYSIS ON CFL STABILITY CONDITION}

Consider the advection equation (6) on the computational domain $x \in[-\pi, \pi]$ with constant speed $u=1$. Provided $\bar{P}_{i-1 / 2}^{n}$ and $\bar{V}_{i}^{n}$ is known, using the one-dimensional algorithm described in Section 2, the updating formulations are written as

$$
\begin{aligned}
& \bar{P}_{i-\frac{1}{2}}^{n+1}=\sum_{p=i-2, q=1}^{p=i, q=3} \gamma_{E}^{q} \bar{P}_{p-\frac{1}{2}}^{n}+\sum_{p=i-2, q=5}^{p=i-1, q=6} \gamma_{E}^{q} \bar{P}_{p}^{n}, \\
& \bar{P}_{i}^{n+1}=\sum_{p=i-2, q=1}^{p=i+1, q=4} \gamma_{C}^{q} \bar{P}_{p-\frac{1}{2}}^{n}+\sum_{p=i-2, q=5}^{p=i, q=7} \gamma_{C}^{q} \bar{P}_{p}^{n},
\end{aligned}
$$


Table A1. The weighting coefficients of the updating formulation.

\begin{tabular}{|c|c|c|c|c|c|c|c|c|}
\hline & \multicolumn{4}{|c|}{ 3-point formula } & \multicolumn{4}{|c|}{ 5-point formula } \\
\hline & \multicolumn{2}{|c|}{$\mathrm{CFL}=0.8$} & \multicolumn{2}{|c|}{$\mathrm{CFL}=1.2$} & \multicolumn{2}{|c|}{$\mathrm{CFL}=1.2$} & \multicolumn{2}{|c|}{$\mathrm{CFL}=1.8$} \\
\hline & $\gamma_{E}$ & $\gamma_{C}$ & $\gamma_{E}$ & $\gamma_{C}$ & $\gamma_{E}$ & $\gamma_{C}$ & $\gamma_{E}$ & $\gamma_{E}$ \\
\hline $\bar{P}_{i-\frac{5}{2}}^{n}$ & 0 & 0 & $-\frac{3}{25}$ & $-\frac{3}{500}$ & $-\frac{3}{25}$ & $\frac{33}{2500}$ & $\frac{12}{25}$ & $-\frac{3}{25}$ \\
\hline $\bar{P}_{i-\frac{3}{2}}^{n}$ & $\frac{12}{25}$ & $-\frac{11}{125}$ & $\frac{12}{25}$ & $\frac{117}{500}$ & $\frac{12}{25}$ & $\frac{1017}{2500}$ & $-\frac{3}{25}$ & $\frac{36}{125}$ \\
\hline $\bar{P}_{i-\frac{1}{2}}^{n}$ & $-\frac{3}{25}$ & $\frac{2}{5}$ & 0 & $\frac{23}{500}$ & 0 & $-\frac{253}{2500}$ & 0 & $\frac{17}{125}$ \\
\hline $\bar{P}_{i+\frac{1}{2}}^{n}$ & 0 & $\frac{1}{125}$ & 0 & $\frac{23}{500}$ & 0 & $\frac{3}{2500}$ & 0 & $\frac{2}{125}$ \\
\hline $\bar{P}_{i-2}^{n}$ & 0 & 0 & $\frac{16}{25}$ & $\frac{4}{125}$ & $\frac{16}{25}$ & $-\frac{44}{625}$ & $\frac{16}{25}$ & $\frac{106}{125}$ \\
\hline $\bar{P}_{i-1}^{n}$ & $\frac{16}{25}$ & $\frac{92}{125}$ & 0 & $\frac{4}{5}$ & 0 & $\frac{468}{625}$ & 0 & $-\frac{11}{125}$ \\
\hline $\bar{P}_{i}^{n}$ & 0 & $-\frac{7}{125}$ & 0 & $-\frac{19}{125}$ & 0 & $\frac{1}{625}$ & 0 & $-\frac{2}{25}$ \\
\hline
\end{tabular}

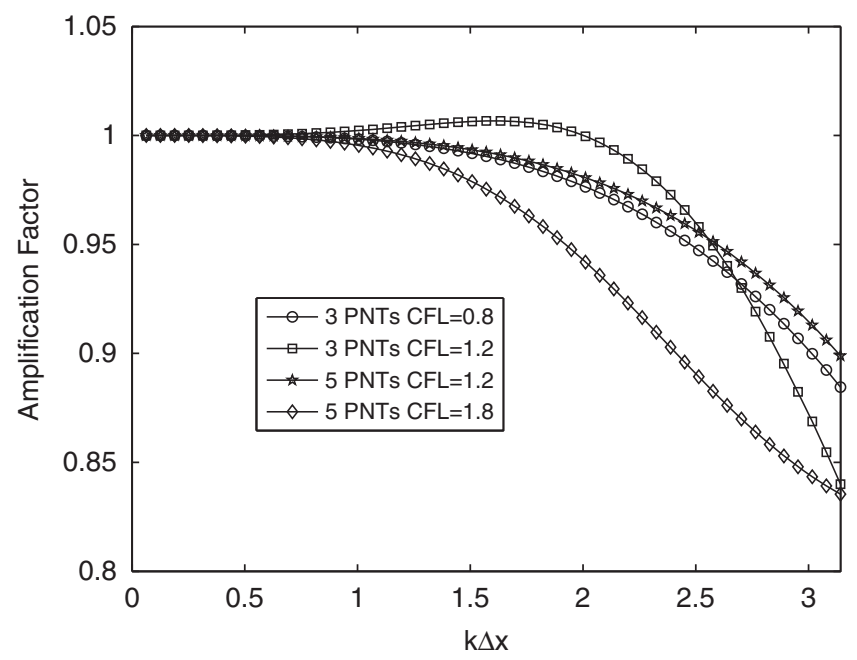

Figure A1. The amplitude gain of different sampling schemes at different CFL numbers.

where $\bar{P}_{i}^{n}$ and $\bar{P}_{i}^{n+1}$ are auxiliary PVs which are calculated using reconstruction polynomial (3) based on known PV and VIA moments with $\tilde{x}=\Delta x / 2$ and $\mathrm{CFL}<2$ is considered here. Hence, the control volume $\mathscr{C}_{i-2}, \mathscr{C}_{i-1}$ and $\mathscr{C}_{i}$ are involved in updating formulations (A1).

Then we use the Von Neumann method to check the CFL stability condition of this linear problem. Updating formulations (A1) are transformed into spectral space. According to the discrete Fourier transformation, we have

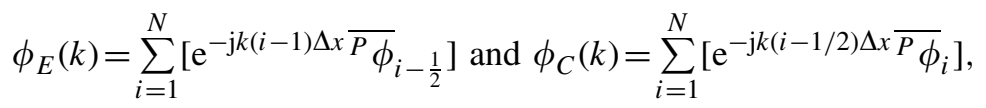

where $\phi_{E}$ and $\phi_{C}$ are representations in spectral space of the PV moments $\bar{P}_{i-1 / 2}$ defined at the endpoints and $\bar{P}_{i}$ defined at the midpoints of the control volumes. $N$ is the number of the control volumes and $\mathrm{j}=\sqrt{-1}$. 
Multiplying the first equation of (A1) with $\mathrm{e}^{-\mathrm{j} k(i-1) \Delta x}$ and the second equation of (A1) with $\mathrm{e}^{-\mathrm{j} k(i-1 / 2) \Delta x}$, and summing (A1) from $i=1$ to $N$, we obtain

$$
\left[\begin{array}{l}
\phi_{E}^{n+1}(k) \\
\phi_{C}^{n+1}(k)
\end{array}\right]=\mathbf{A}\left[\begin{array}{l}
\phi_{E}^{n}(k) \\
\phi_{C}^{n}(k)
\end{array}\right],
$$

where matrix $\mathbf{A}$ is the amplification factor and written as

$$
\mathbf{A}=\left[\begin{array}{cc}
\sum_{p=1}^{3} \gamma_{E}^{p} \mathrm{e}^{-\mathrm{j}(3-p) k \Delta x} & \sum_{p=5}^{6} \gamma_{E}^{p} \mathrm{e}^{-\mathrm{j}\left(\frac{13}{2}-p\right) k \Delta x} \\
\sum_{p=1}^{4} \gamma_{C}^{p} \mathrm{e}^{-\mathrm{j}\left(\frac{7}{2}-p\right) k \Delta x} & \sum_{p=5}^{7} \gamma_{C}^{p} \mathrm{e}^{-\mathrm{j}(7-p) k \Delta x}
\end{array}\right] .
$$

Equation (A3) is the representation of the updating formulation (A1) in spectral space; the spectral radius of matrix A dominates the stability of the numerical schemes.

We consider two algorithms for numerical integration of the flux. Besides the 3-point Simpson's rule (12), 5-point Simpson's rule,

$$
\begin{aligned}
& \alpha_{1}=0, \quad \alpha_{2}=\frac{1}{4}, \quad \alpha_{2}=\frac{1}{2}, \quad \alpha_{4}=\frac{3}{4}, \quad \alpha_{5}=1, \\
& \beta_{1}=\beta_{5}=\frac{7}{90}, \quad \beta_{2}=\beta_{4}=\frac{16}{45}, \quad \beta_{3}=\frac{2}{15}
\end{aligned}
$$

can be adopted for simulations with larger CFL numbers.

The coefficients of updating formulations with different integration algorithms and CFL numbers are given in Table A1. The spectral radius of different cases are plotted in Figure A1. According to the results of the Von Neumann analysis, using 3-point formulation for integration of the flux restricts the $\mathrm{CFL}<1$ to assure the stability of the numerical model. Sampling more points along the trajectory allows larger stable CFL number, but more computational cost are involved at each time step. The proper integration algorithm can be selected on a trade-off base between the overall cost and the accuracy of numerical solution.

\section{ACKNOWLEDGEMENTS}

This work is supported by National Natural Science Foundation of China under grant numbers 10902116, 10852001 and 40805045, and Chinese Academy of Sciences under grant number KJCX2-YW-L04.

\section{REFERENCES}

1. Staniforth A, Côté J. Semi-Lagrangian integration schemes for atmospheric models-a review. Monthly Weather Review 1991; 119:2206-2223.

2. Yabe T, Aoki T. A universal solver for hyperbolic equations by cubic polynomial interpolation.1. One-dimensional solver. Computer Physics Communications 1991; 66:219-232.

3. Yabe T, Xiao F, Utsumi T. The constrained interpolation profile method for multiphase analysis. Journal of Computational Physics 2001; 169:556-593.

4. Yabe T, Tanaka R, Nakamura T, Xiao F. An exactly conservative semi-Lagrangian scheme (CIP-CSL) in one dimension. Monthly Weather Review 2001; 129(2):332-344.

5. Xiao F, Yabe T. Completely conservative and oscillationless semi-Lagrangian schemes for advection transportation. Journal of Computational Physics 2001; 170(2):498-522.

6. Nakamura T, Tanaka R, Yabe T, Takizawa K. Exactly conservative semi-Lagrangian scheme for multi-dimensional hyperbolic equations with directional splitting technique. Journal of Computational Physics 2001; 174:171-207.

7. Xiao F, Yabe T, Peng XD, Kobayashi H. Conser and oscillation-less atmospheric transport schemes based on rational functions. Journal of Geophysical Research 2002; 107:4609.

8. Takizawa K, Yabe T, Nakamura T. Multi-dimensional semi-Lagrangian scheme that guarantees exact conservation. Computer Physics Communications 2002; 148:137-159.

9. Xiao F. Unified formulation for compressible and incompressible flows by using multi-integrated moments I: one-dimensional inviscid compressible flow. Journal of Computational Physics 2004; 195(2):629-654. 
10. Xiao F, Akoh R, Ii S. Unified formulation for compressible and incompressible flows by using multi-integrated moments II: Multi-dimensional version for compressible and incompressible flows. Journal of Computational Physics 2006; 213(1):31-56.

11. Nair RD, Machenhauer B. The mass-conservative cell-integration semi-Lagrangian advection scheme on the sphere. Monthly Weather Review 2002; 130:649-667.

12. Lauritzen PH, Nair RD, Ullrich PA. A conservative semi-lagrangian multi-tracer transport scheme (CSLAM) on cubed-sphere grid. Journal of Computational Physics 2010; 229:1401-1424.

13. Sadourny R, Arakawa A, Mintz Y. Integration of the nondivergent barotropic vorticity equation with an icosahedralhexagonal grid for the sphere. Monthly Weather Review 1968; 96(6):351-356.

14. Williamson DL. Integration of the barotropic vorticity equation on a spherical geodesic grid. Tellus 1968; 20(4):642-653.

15. Sadourny R. Conservative finite-difference approximations of the primitive equations on quasi-uniform spherical grids. Monthly Weather Review 1972; 100(2):136-144.

16. McGregor JL. Semi-Lagrangian advection on conformal-cubic grids. Monthly Weather Review 1996; 124(6): 1311-1322.

17. McGregor JL. Semi-Lagrangian advection on a cubic gnomonic projection of the sphere. Atmosphere-Ocean 1997; Memorial Volume:153-169.

18. Nair RD, Thomas SJ, Loft RD. A discontinuous Galerkin global shallow water model. Monthly Weather Review 2005; 133(4):876-887.

19. Nair RD, Thomas SJ, Loft RD. A discontinuous Galerkin transport scheme on the cubed sphere. Monthly Weather Review 2005; 133(4):827-841.

20. Rancic M, Purser RJ, Mesinger F. A global shallow-water model using an expanded spherical cube: gnomonic versus conformal coordinates. Quarterly Journal of the Royal Meteorological Society 1996; 122(532):959-982.

21. Ronchi C, Iacono R, Paolucci PS. The 'Cubed Sphere': a new method for the solution of partial differential equations in spherical geometry. Journal of Computational Physics 1996; 124(1):93-114.

22. Rossmanith JA. A wave propagation method for hyperbolic systems on the sphere. Journal of Computational Physics 2006; 213(2):629-658.

23. Thomas SJ, Loft RD. Semi-implicit spectral element atmospheric model. Journal of Scientific Computing 2002; 17(1-4):339-350.

24. Adcroft A, Campin JM, Hill C, Marshall J. Implementation of an atmosphere-ocean general circulation model on expended spherical cube. Monthly Weather Review 2004; 132(12):2845-2863.

25. Putman WP, Lin SJ. Finite-volume transport on various cubed-sphere grids. Journal of Computational Physics 2007; 227:55-78.

26. Chen CG, Xiao F. Shallow water model on cubed sphere by muti-moment finite volume method. Journal of Computational Physics 2008; 227:5019-5044.

27. Ii S, Xiao F. CIP/multi-moment finite volume method for Euler equations: a semi-Lagrangian characteristic formulation. Journal of Computational Physics 2007; 222(2):849-871.

28. Bermejo R, Staniforth A. The conversion of the semi-Lagrangian advection schemes to quasi-monotone schemes. Monthly Weather Review 1992; 120:2622-2632.

29. Williamson DL, Drake JB, Hack JJ, Jakob R, Swarztrauber PN. A standard test set for numerical approximations to the shallow water equations in spherical geometry. Journal of Computational Physics 1992; 102(1):211-224.

30. Jiang GS, Shu CW. The efficient implementation of weighted ENO schemes. Journal of Computational Physics 1996; 126:202-228.

31. Nair RD, Scroggs JS, Semazzi FH. A forward-trajectory global semi-lagrangian transport scheme. Journal of Computational Physics 2003; 190:275-294.

32. Giraldo FX. Trajectory calculations for spherical geodestic grids in cartesian space. Monthly Weather Review 1999; 127:1651-1662.

33. Zerroukat M, Wood N, Staniforth A. A monotonic and positive-definite filter for a semi-Lagrangian Inherently conserving and efficient (SLICE) scheme. Quarterly Journal of the Royal Meteorological Society 2005; 131: 2923-2936.

34. Nair RD, Côté J, Staniforth A. Cascade interpolation for semi-Lagrangian advection over the sphere. Quarterly Journal of the Royal Meteorological Society 1999; 125(556):1445-1468.

35. Nair RD, Jablonowski C. Moving vortices on the sphere: a test case for horizontal advection problems. Monthly Weather Review 2008; 136:699-711. 